\title{
Manifestaciones neurológicas atípicas del síndrome de reconstitución inmunológico por infección con el virus de inmunodeficiencia humana, reporte de dos casos
}

\author{
Francisco Bernal-Cano ${ }^{1}$, Jorge Oswaldo Suárez ${ }^{1}$, Carlos Arturo Álvarez ${ }^{1}$, Ellen Lowenstein ${ }^{1}$, \\ Sandra Liliana Valderrama ${ }^{1}$, Carlos Hernando Gómez ${ }^{1}$, José Roberto Támara ${ }^{1}$, \\ IVÁN RAMIRO TENORIO ${ }^{1}$
}

\section{Resumen}

El síndrome inflamatorio de reconstitución inmunológica en caso de infección por el virus de inmunodeficiencia humana (VIH) es una entidad clínica rara, que se manifiesta con la activación de procesos infecciosos oportunistas que se encontraban estables.

Presentamos dos casos de este síndrome con compromiso del sistema nervioso central: síndrome de movimientos anormales y periférico, y síndrome de Guillain-Barré.

Palabras clave: VIH, síndrome de inmunodeficiencia humana, síndrome inflamatorio de reconstitución inmunológica, terapia HAART, coreoatetosis, polineuropatía desmielinizante inflamatoria aguda, síndrome de Guillain-Barré.

\section{Title}

Atypical neurological manifestations of immunologic reconstitution syndrome in the context of HIV infection, a report of two case studies

\begin{abstract}
Immune reconstitution inflammatory syndrome in the context of severe immunosupression secondary to human immunodeficiency virus (HIV) is a clinical entity manifested by a paradoxical clinical impairment due to an activation of infectious opportunistic processes previously under immune surveillance.

We illustrate two case reports of immune reconstitution inflammatory syndrome with atypical central nervous system involvement (choreoathetosis) and acute inflammatory demyelinating polineuropathy (Guillain-Barré syndrome).

Key words: HIV, acquired immunodeficiency syndrome, immune reconstitution inflammatory syndrome, antiretroviral therapy, highly active, movement disorders, GuillainBarré syndrome.
\end{abstract}

1 Unidad de Infectología. Hospital Universitario San Ignacio. Bogotá D.C., Colombia. 


\section{Presentación de los casos}

\section{Caso 1}

Se trata de un paciente de sexo masculino, de 38 años de edad con diagnóstico de infección por virus de inmunodeficiencia humana (VIH) en abril de 2007, con conteo de linfocitos T CD4+ de 82 células/ $\mu 1$ y carga viral para VIH en suero mayor de 100.000 copias/ml. Inició terapia HAART (Highly Active Antiretroviral Therapy) con zidovudina, lamivudina y efavirenz, un año después de la confirmación de su diagnóstico.

En julio de 2008 consultó a urgencias de la Clínica Partenón por un cuadro clínico de dos semanas de evolución de movimientos involuntarios del miembro superior izquierdo. En el examen físico de ingreso se observó coreoatetosis de todo el hemicuerpo izquierdo.

Se diagnosticó un síndrome de movimientos anormales y se solicitaron los siguientes exámenes: hemograma, electrolitos, perfil hepático, renal y tiroideo, radiografía de tórax, tomografía computadorizada (TC) simple de cráneo, prueba de reagina plasmática rápida, antiestreptolisinas, ceruloplasmina, cobre y serología para Toxoplasma gondii, todos sin resultados anormales.

El paciente fue hospitalizado para completar su estudio e iniciar tratamiento. Se practicó una resonancia magnética ( $\mathrm{RM})$ cerebral simple en la que se observaron varias lesiones hiperintensas en el putamen, el globo pálido, la cápsula interna y el tálamo del lado derecho, con compromiso adicional de aspecto inespecífico de la circunvolución central (figura 1).

Se hizo una punción lumbar con líquido cefalorraquídeo claro, presión de apertura y análisis citoquímico dentro de límites normales; las tinciones de Gram, de Zielh-Neelsen y de tinta china, y el cultivo para hongos, no mostraron presencia de patógenos. Además, se solicitó detección del antígeno para Criptococcus neoformans por látex, prueba serológica para la sífilis (VDRL), reacción en cadena de la polimerasa (PCR) para la detección del ADN de citomegalovirus, herpes simple de tipos 1 y 2 y bacilo tuberculoso, los cuales fueron resultados negativos.

Durante la estancia hospitalaria, se solicitó un electroencefalograma, en el que se observó un trazado normal.

Se inició tratamiento sintomático con $5 \mathrm{mg}$ de haloperidol por vía oral, cada 12 horas. En vista de que no presentó empeoramiento de su cuadro clínico, fue dado de alta con el mismo esquema antirretroviral y se solicitó un nuevo conteo de linfocitos T CD4+ y carga viral para VIH en suero.

Al término de tres semanas, el paciente asistió a control y reportó disminución de la amplitud de los movimientos coreicos. La detección de 
ADN para citomegalovirus, herpes simple de tipos 1 y 2 y bacilo tuberculoso en líquido cefalorraquídeo fue negativa; el recuento de linfocitos $\mathrm{T}$ CD4+ fue de 245 células/ $\mu 1$ y la carga viral para VIH en suero fue menor de 40 copias $/ \mathrm{ml}$.

En esta ocasión, el examen neurológico sólo mostró discreta rigidez apendicular de ambos lados. Se diagnosticó un síndrome inflamatorio de reconstitución inmunológica que se manifestó clínicamente por un síndrome extrapiramidal, actualmente modificado por la toma de haloperidol.

En vista de que el paciente no presentó otro tipo de complicaciones y experimentó mejoría parcial de los síntomas iniciales, se decidió suspender la administración de haloperidol y continuar con el mismo tratamiento antirretroviral por tiempo indefinido. Se solicitó, además, una nueva RM cerebral simple.

Cuatro semanas más tarde asistió a control y refirió mejoría de los síntomas; además, en el nuevo estudio de RM se observaba disminución del número y el tamaño de las lesiones hiperintensas, en comparación con el estudio previo (figura 2). Desde entonces, el paciente ha estado asintomático y su conteo promedio de linfocitos $\mathrm{T}$ CD4+ se ha mantenido alrededor de 200 células/ $\mu 1$.

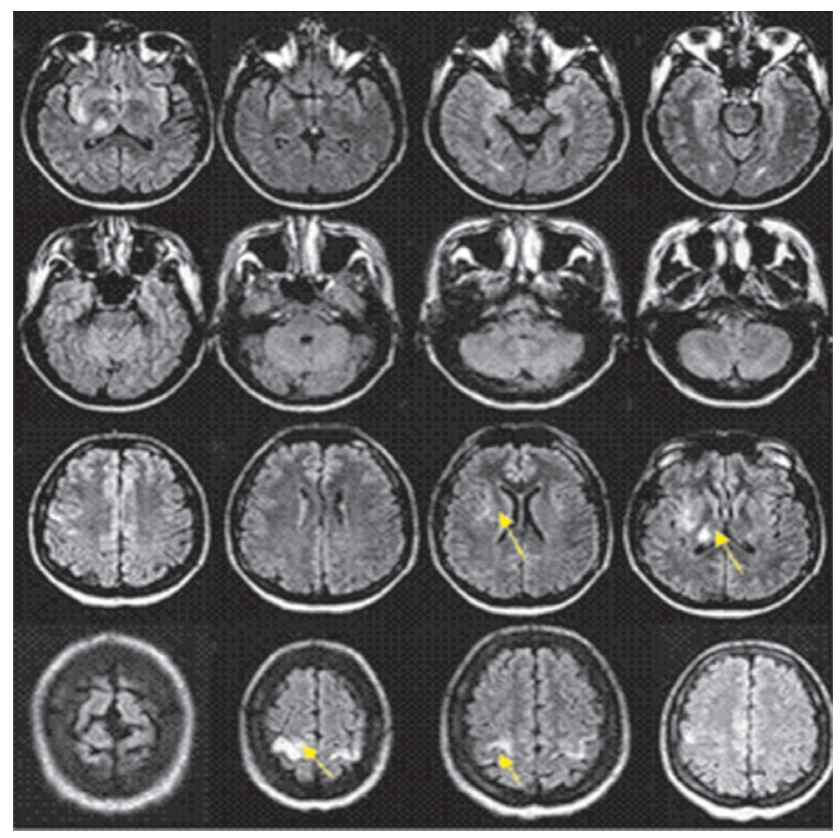

Figura 1. Resonancia magnética cerebral secuencia FLAIR (Fluid Attenuated Inversion Recovery). Se aprecian zonas hiperintensas en la región ganglio basal derecha, con extensión al tálamo y compromiso adicional de la circunvolución central (flechas). 


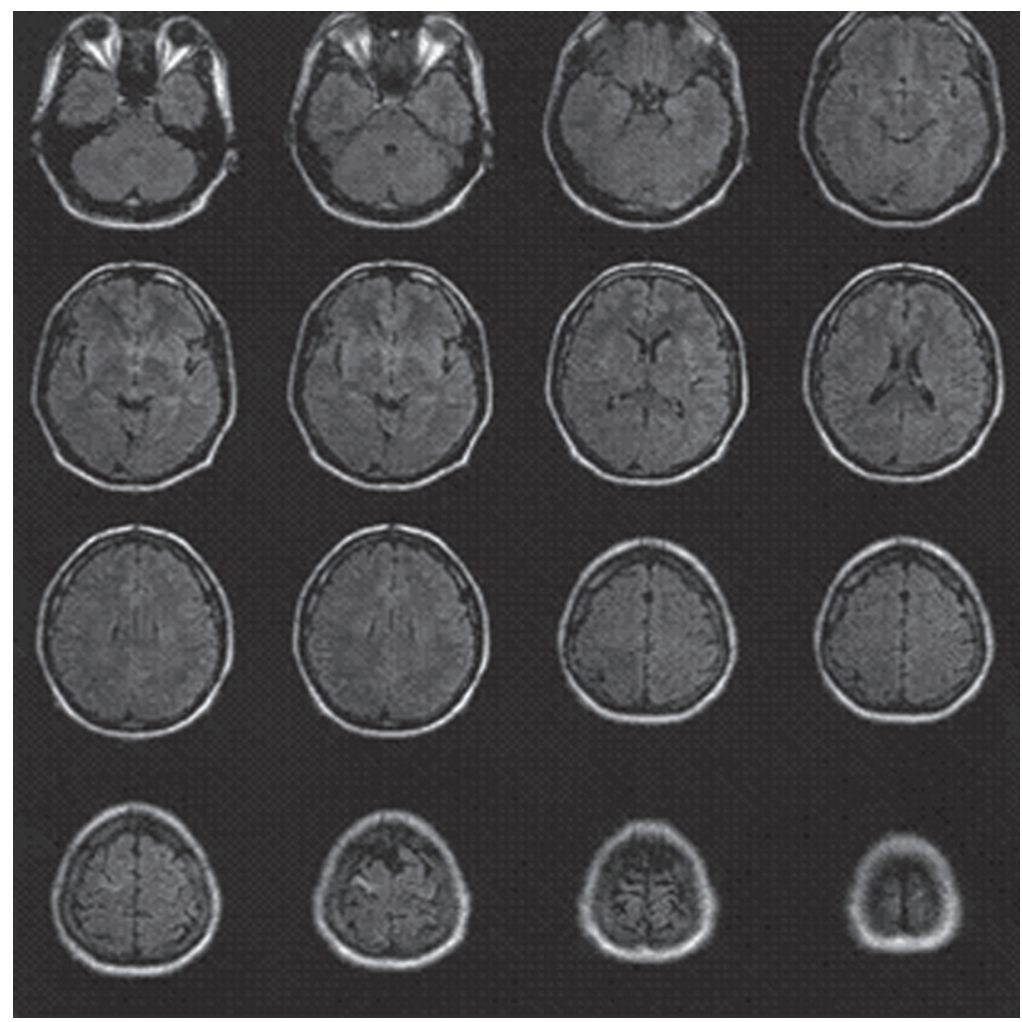

Figura 2. Resonancia magnética cerebral secuencia FLAIR tomada cuatro semanas después, en el que se aprecia disminución del número de lesiones hiperintensas que corresponde con la mejoría clínica del paciente.

\section{Caso 2.}

Se trata de un paciente de sexo masculino de 46 años de edad con diagnóstico de infección por VIH en febrero de 2008, en el contexto de una neumonía por Pneumocystis jiroveci, conteo de linfocitos T CD4+ de 49 células/ $\mu 1$ y carga viral para VIH mayor de 100.000 copias/ml; se le inició tratamiento antirretroviral con zidovudina, lamivudina y efavirenz en la segunda semana de marzo de ese año.
Consultó a urgencias del Hospital Universitario San Ignacio por un cuadro de seis días de evolución de pérdida de la fuerza en las cuatro extremidades, con dificultad para la marcha y la manipulación de objetos.

En el examen físico de ingreso sólo se encontró cuadriparesia proximal y distal de 4 sobre 5 , con ausencia de los reflejos miotendinosos en los miembros inferiores, sin déficit sensitivo. Se diagnosticó un síndrome de 
debilidad aguda, secundario a probable polineuropatía desmielinizante inflamatoria (síndrome de Guillain-Barré).

Se solicitaron los siguientes exámenes: hemograma, electrolitos, perfil hepático y renal, función tiroidea, vitamina B12, ácido fólico, reagina plasmática rápida y ácido láctico, con resultados por fuera del rango patológico. El conteo de linfocitos T CD4+ tomado dos semanas antes fue de 221 células/ $\mu$ y la carga viral para VIH en suero fue menor de 40 copias $/ \mathrm{ml}$.

Se decidió hospitalizar con el fin de observar la progresión de los síntomas y hacer estudio del líquido cefalorraquídeo. En el análisis citoquímico se reportó: células, 0; proteínas, 157 $\mathrm{mg} / \mathrm{dl}$, y glucosa, $54 \mathrm{mg} / \mathrm{dl}$; los cultivos fueron negativos; el látex para $C$. neoformans y el VDRL no fueron reactivos; la detección de ADN viral por PCR para citomegalovirus fue negativa y la carga viral para VIH fue menor de 200 copias $/ \mathrm{ml}$.

Durante su hospitalización no experimentó empeoramiento de los síntomas y se solicitó estudio de neuroconducción y electromiografía de las cuatro extremidades. En el estudio se encontró reducción generalizada de las velocidades motoras de conducción con disminución de las ondas $\mathrm{F}$ y leve compromiso del reclutamiento de las unidades motoras.

Con estos resultados se confirmó el diagnóstico de una polineuropatía desmielinizante inflamatoria aguda, por síndrome inflamatorio de reconstitución inmunológica.

Se suspendió temporalmente el tratamiento antirretroviral por el riesgo de que presentara progresión de los síntomas y, al término de la tercera semana de haberse hecho el diagnóstico, el paciente presentó mejoría de la fuerza en los miembros inferiores.

Se reinició el tratamiento antirretroviral sin modificación y actualmente, después la terapia física, presenta un examen neurológico dentro de límites normales, con un conteo de linfocitos T CD4+ por encima de 200 células/ $\mu 1$ y una carga viral para VIH en suero en rango de no detección.

\section{Discusión}

Con el advenimiento de la terapia HAART se ha generado una importante disminución de la frecuencia de las infecciones oportunistas en los pacientes con infección por VIH, con lo cual se ha conseguido una reducción significativa de la tasa de mortalidad asociada a estas alteraciones. Sin embargo, un subgrupo de pacientes que reciben terapia HAART puede presentar un deterioro paradójico a pesar de lograr un control satisfactorio sobre la replicación del VIH y un aumento en el conteo de linfocitos T CD4+.

Este estado clínico, conocido como síndrome inflamatorio de reconstitución inmunológico, es el resultado de una respuesta inflamatoria excesiva dirigi- 
da, generalmente, a los antígenos de los procesos infecciosos en fase de remisión o inactivos y, usualmente, ocurre cuando el conteo de linfocitos T CD4+ de base se encuentra por debajo de 100 células/ $\mu 1$. Entre las manifestaciones clínicas del síndrome inflamatorio de reconstitución inmunológica sobresalen las exacerbaciones de toxoplasmosis, tuberculosis, linfadenitis secundaria al complejo Mycobacterium avium, criptococosis meníngea $\mathrm{y}$ leucoencefalopatía multifocal progresiva, entre otras $[1,2]$.

De acuerdo con la literatura, alrededor de $25 \%$ a $35 \%$ de los pacientes con infección por VIH que inician terapia HAART desarrollan un síndrome inflamatorio, de reconstitución inmunológica el cual se presenta en la mayoría de los casos en los primeros 60 días de tratamiento[3-5].

Infortunadamente, no existe en el momento un consenso estandarizado sobre la definición y el diagnóstico de este síndrome[6]. Sin embargo, las definiciones propuestas por grupos de expertos incluyen los siguientes criterios[7-9]:

a. Caso confirmado de infección por VIH.

b. Asociación temporal entre el desarrollo de síndrome inflamatorio de reconstitución inmunológica y el inicio de la terapia HAART. c. Respuesta clara del huésped al inicio de la terapia HAART, representada por un incremento del conteo de linfocitos T CD4 y supresión de la carga viral del VIH en suero, al menos $1 \log _{10}$.

d. Deterioro clínico caracterizado por un proceso inflamatorio.

e. Exclusión de otras causas que pueden generar un cuadro clínico similar.

f. Otras causas tales como resistencia o toxicidad por la terapia HAART, mala absorción de los medicamentos, falta de seguimiento del tratamiento, sobreinfección por otros patógenos, tratamiento antimicrobiano inadecuado del proceso infeccioso preexistente y desarrollo de complicaciones no infecciosas.

En el primer caso, se expone un claro compromiso de la integridad del sistema nervioso central manifestado por un síndrome extrapiramidal en presencia de una reconstitución inmunológica al corto tiempo de haber iniciado la terapia HAART. Sin embargo, con el abordaje diagnóstico empleado no se detectó ningún proceso infeccioso concomitante con el VIH, lo cual es inusual en el síndrome inflamatorio de reconstitución inmunológica, pero no lo descarta. 
La presencia de movimientos anormales asociada a la infección por VIH se ha reportado en la literatura en los periodos de seroconversión y en el estadio de inmunosupresión profunda, cuando el curso clínico se modifica con el inicio de la terapia HAART[10]. No obstante, hasta el momento no hay reportes sobre su aparición con el síndrome inflamatorio de reconstitución inmunológica y se presume que el mecanismo por el cual los ganglios basales y sus conexiones son afectados por la infección por VIH se explica por un efecto citopático por el mismo virus o por un fenómeno inmunológico de aparición tardía en el huésped, ambas hipótesis actualmente en discusión[10, $11]$.

En el caso de la encefalopatía por $\mathrm{VIH}$, se ha demostrado un daño grave del sistema dopaminérgico manifestado por una reducción de los niveles del ácido homovalínico en el líquido cefalorraquídeo y por la pérdida neuronal en el globo pálido. Estos hallazgos no sólo explican la existencia de un síndrome de movimientos anormales o de enlentecimiento psicomotor, sino la importante sensibilidad a dosis bajas de neurolépticos que se manifestó por extrapiramidalismo[12].

Nuestro paciente no sólo experimentó mejoría de los síntomas al suspender temporalmente la terapia HAART en conjunto con la administración sinérgica de haloperidol, sino que presentó rigidez apendicular, lo cual está acorde con lo expuesto anteriormente.

El efecto terapéutico de los antipsicóticos en la coreoatetosis no es del todo claro. Sin embargo, se presume que está mediado por el bloqueo dopaminérgico D2 que muestra en la mayoría de los casos una respuesta clínica variable, por lo cual se han propuesto otros abordajes terapéuticos tales como el uso de anticonvulsivos, reserpina o tetrabenazina, entre otros, cuyo mecanismo de acción es aún desconocido[13].

En el segundo caso se expone un compromiso del sistema nervioso periférico manifestado por un síndrome de Guillain-Barré, también al corto tiempo de haber iniciado la terapia HAART. Es probable que el rápido aumento del conteo de linfocitos $\mathrm{T}$ CD4+ y CD8+ haya desencadenado una respuesta inmunitaria dirigida inicialmente a los antígenos del VIH que, de manera cruzada, afectó la integridad de la mielina del nervio periférico[14]. Este fenómeno puede explicarse por el papel de los linfocitos $\mathrm{T}$ en la selección natural de conjuntos de anticuerpos autorreactivos, cuya expresión de inmunoglobulinas hacia algunos antígenos del huésped depende del número de linfocitos $\mathrm{T}$ CD4+[15].

El síndrome de Guillain-Barré se ha descrito en pacientes con infección por VIH durante el periodo de seroconversión. Sin embargo, algunos 
casos se han presentado en presencia de inmunosupresión profunda. En este último caso, se presume que el mecanismo de acción sea explicado por un aumento de la actividad de los linfocitos $\mathrm{T}$ y $\mathrm{B}$ en presencia de linfopenia inducida por la alteración de la homeostasis de las células $T$, lo que permite una pérdida de la autotolerancia antigénica, que desencadenaría un fenómeno autoinmunitario en el paciente inmunosuprimido[16].

Hasta el momento no hay estudios o guías de manejo que provean suficientes pruebas respecto al tratamiento del síndrome inflamatorio de reconstitución inmunológica. Con base en varios reportes de casos, podemos concluir que existen maneras de prevenir el desarrollo de este síndrome, las cuales dependen de la tamización de infecciones oportunistas antes de considerar el uso de la terapia HAART. Cuando el conteo de linfocitos T CD4+ sea menor de 100 células/ $\mu 1$, algunos autores sugieren el inicio de la terapia HAART una vez se haya iniciado el tratamiento específico para las infecciones concomitantes detectadas, con el fin de disminuir la carga antigénica que pudiera desencadenar el síndrome. Cuando se diagnostica síndrome inflamatorio de reconstitución inmunológica las opciones de tratamiento dependen del grado de compromiso clínico del paciente; si el síndrome está relacionado con un daño irreversible, tal como retinitis por citomegalovirus, la terapia HAART en teoría podría suspenderse temporalmente e iniciarse tratamiento específico para el proceso infeccioso subyacente, de lo contrario, podría continuarse la terapia HAART.

No existe un tratamiento definitivo para el síndrome inflamatorio de reconstitución inmunológica con compromiso del sistema nervioso central y es incierto si la suspensión temporal del tratamiento antirretroviral o el uso de esteroides modifiquen el curso de la enfermedad[17]. Este abordaje terapéutico surge al inferir un posible efecto de la suspensión del estímulo inmunológico de la terapia HAART sobre la magnitud del compromiso inflamatorio del parénquima cerebral y la supresión de una respuesta potencialmente citotóxica que desencadene muerte celular. Algunos autores sugieren el uso de esteroides ante la inminencia de deterioro neurológico secundario a un síndrome de hipertensión endocraneana que conlleve a un cuadro de herniación cerebral[17, 18].

\section{Conclusión}

En ausencia de más estudios, poco se conoce acerca del síndrome inflamatorio de reconstitución inmunológica. Es probable que en los próximos años la incidencia de este síndrome aumente, especialmente entre los pacientes con infección por VIH que cursen con una alta carga antigénica y bajo conteo de linfocitos T CD4+. Infortunadamente, 
la fisiopatología del síndrome inflamatorio de reconstitución inmunológica aún genera muchos interrogantes, debido a que los biomarcadores para su diagnóstico y predicción no se conocen del todo. Es necesaria la realización de más estudios, con el fin de validar y refinar los criterios diagnósticos de esta entidad.

\section{Agradecimientos}

Especial agradecimiento a María Clara Castro y a Marcia Ávila Téllez por su ayuda en la elaboración de este manuscrito. De igual manera, al doctor Julio César Castellanos, director del Hospital Universitario San Ignacio por su apoyo a la consulta de NeuroInfectología.

\section{Bibliografía}

1. Shelburne SA 3rd, Hamill RJ. The immune reconstitution inflammatory syndrome. AIDS Rev. 2003;5:67-79.

2. Venkataramana A, Pardo CA, McArthur JC, Kerr DA, Irani DN, Griffin JW, et al. Immune reconstitution inflammatory syndrome in the CNS of HIV-infected patients. Neurology. 2006;67:383-8.

3. Tsang CS, Samaranayake LP. Immune reconstitution inflammatory syndrome after highly active antiretroviral therapy: A review. Oral Dis. 2010; 16(3):248-56.

4. French MA, Lenzo N, John M, Mallal SA, McKinnon EJ, James IR, et al. Immune restoration disease after the treatment of immunodeficient HIV- infected patients with highly active antiretroviral therapy. HIV Med. 2000;1:107-15.

5. Narita M, Ashkin D, Hollender ES, Pitchenik AE. Paradoxical worsening of tuberculosis following antiretroviral therapy in patients with AIDS. Am J Respir Crit Care Med. 1998;158:157-61.

6. Haddow LJ, Easterbrook PJ, Mosam A, Khanyile NG, Parboosing R, Moodley $P$. Defining immune reconstitution inflammatory syndrome: Evaluation of expert opinion versus 2 case definitions in a South African cohort. Clin Infect Dis. 2009;49:1424-32.

7. Shelburne SA 3rd, Hamill RJ, Rodríguez-Barradas MC, Greenberg $\mathrm{SB}$, Atmar RL, Musher DW, et al. Immune reconstitution inflammatory syndrome: Emergence of a unique syndrome during highly active antiretroviral therapy. Medicine. 2002;81:213-27.

8. French MA, Price P, Stone SF. Immune restoration disease after antiretroviral therapy. AIDS. 2004;18:1615-27.

9. Müller M, Wandel S, Colebunders R, Attia S, Furrer H, Egger M. Immune reconstitution inflammatory syndrome in patients starting antiretroviral therapy for HIV infection: A systematic review and meta-analysis. Lancet Infect Dis. 2010;10:251-61.

10. Trocello JM, Blanchet A, Bourdain F, Meyohas MC, Vidailhet M. Resolution of choreic movements associated with HIV encephalitis with anti-retroviral therapy. Rev Neurol (Paris). 2006; 162:89-91.

11. Passarin MG, Alessandrini F, Nicolini GG, Musso A, Gambina G, Moretto G. 
Reversible choreoathetosis as the early onset of HIV-encephalopathy. Neurol Sci. 2005;26:55-6.

12. Tse W, Cersosimo MG, Gracies JM, Morgello S, Olanow CW, Koller W. Movement disorders and AIDS: A review. Parkinsonism Relat Disord. 2004;10:323-34.

13. Cardoso F. HIV-related movement disorders: Epidemiology, pathogenesis and management. CNS Drugs. 2002;16:663-8.

14. Teo EC, Azwra A, Jones RL, Gazzard BG, Nelson M. Guillain-Barré syndrome following immune reconstitution after antiretroviral therapy for primary HIV infection. $J$ HIV Ther. 2007;12:62-3.
15. Stahl D, Lacroix-Desmazes S, Misra N, Karmochkine M, Kaveri SV, Costagliola D, et al. Alterations of selfreactive antibody repertoires in HIV disease: An insight into the role of $\mathrm{T}$ cells in the selection of autoreactive B cells. Immunol Lett. 2005;99:198-208.

16. Brannagan TH 3rd, Zhou Y. HIVassociated Guillain-Barré syndrome. $J$ Neurol Sci. 2003;208:39-42.

17. Bernal-Cano F. El virus de inmunodeficiencia humana VIH y el sistema nervioso. Principios generales. Acta Neurol Colomb. 2008;24:124-41.

18. Berger JR. Steroids for PML-IRIS: A double-edged sword? Neurology. 2009;72:1454-5. 